Empfehlungen von Expertengremien 


\title{
Die Ad-hoc-Empfehlung des Deutschen Ethikrats zur Corona-Pandemie
}

\author{
Eberhard Schockenhoff ${ }^{\dagger}$
}

Nur wenige Wochen nach dem Inkrafttreten der von den staatlichen Stellen beschlossenen Maßnahmen zur Eindämmung der Corona-Pandemie am 27. März 2020 veröffentlichte der Deutsche Ethikrat (DER) eine ausführliche Stellungnahme zu den ethischen Bedingungen, unter denen die von der Regierung verfügten Maßnahmen, die mit schwerwiegenden Grundrechtseingriffen und Belastungen für die Bevölkerung verbunden sind, ethisch gerechtfertigt erscheinen. Zum Zeitpunkt der Publikation der Empfehlungen des Ethikrates lagen bereits ähnliche Stellungnahmen von ausländischen und inländischen medizinischen Fachgesellschaften vor, von denen sich die Überlegungen des Ethikrates teilweise deutlich abheben. Die Stellungnahme erörtert drei unterschiedliche Fragekomplexe, in denen neben der Beantwortung naturwissenschaftlicher (virologischer, endemiologischer und intensivmedizinischer) Aspekte auch rechtliche und ethische Überlegungen anzustellen sind.

Dezidiert lehnt es der DER ab, die Bekämpfung der Pandemie allein an die Wissenschaft zu delegieren. Es wäre nicht nur eine Überforderung naturwissenschaftlicher Erkenntnisse über die Entstehung und den voraussichtlichen Verlauf einer Pandemie, wollte man daraus konkrete Handlungsanweisungen zu ihrer Bekämpfung ableiten. Diese müssen zwar aus naturwissenschaftlicher Sicht begründet und angemessen sein, doch widerspräche es dem Grundgedanken demokratischer Legitimation, weitreichende politische Entscheidungen, die das gesellschaftliche Leben auf lange Sicht verändern, in abgeschotteten Zirkeln wissenschaftlicher Politikberatung mit unklaren Verantwortungsstrukturen zu fällen. Deshalb wiederholt der Ethikrat am Schluss nochmals die Eingangsthese seiner Ermahnung, dass die Spielregeln politischer Entscheidungsfindungen durch das Zusammenspiel der Verfassungsorgane einer repräsentativen Demokratie auch in Zeiten der Corona-Krise nicht außer Kraft gesetzt werden dürfen. "Gerade schmerzhafte Entscheidungen müssen von den Organen getroffen werden, die hierfür durch das Volk mandatiert sind und dementsprechend auch in politischer Verantwortung stehen. Die Corona-Krise ist die Stunde der demokratisch legitimierten Politik.“ (S.7) Zum Zeitpunkt der Veröf- 
fentlichung dieser Stellungnahme setzte dieser Hinweis einen markanten Kontrapunkt zu der nahezu ausschließlichen Fokussierung der medialen Aufmerksamkeit auf das Regierungshandeln der staatlichen Stellen und ihre zu Beginn der Krise reibungslose Koordination. Die hohen Zustimmungswerte zu den die Regierung tragenden Parteien und den wichtigsten Einzelpersönlichkeiten (Bundeskanzlerin, Gesundheitsminister, einzelne Ministerpräsidenten) signalisierten aber auch, dass ein Großteil der Bevölkerung - und damit des demokratischen Souveräns und Trägers der letzten politischen Verantwortung - mit den getroffenen Maßnahmen einverstanden war.

Im Einzelnen berühren die rechtlichen und ethischen Erörterungen der Stellungnahme drei Bereiche: erstens die ethische Legitimation der Gesamtstrategie eines weitgehenden gesellschaftlichen Lockdowns, zweitens die Notfallregeln einer aufgrund von Unterkapazitäten erforderlichen Priorisierung einzelner Patienten bei medizinischen Behandlungsentscheidungen und drittens die erforderlichen Öffnungsperspektiven, die eine Renormalisierung des gesellschaftlichen, wirtschaftlichen und kulturellen Lebens ermöglicht.

\section{Die Rechtfertigung der anfänglichen Lockdown-Strategie}

Was die Rechtfertigung der betroffenen Grundentscheidung, das gesamte gesellschaftliche Leben weitgehend „herunterzufahren“, anbelangt, um ein exponentielles Anwachsen der Anzahl infizierter Personen zu vermeiden, betont der DER, dass diese Vorgehensweise in der dramatischen Anfangsphase der Pandemie rechtlich und ethisch vertretbar oder sogar geboten erschien. Dies gilt auch für die „rigorosen, massiv und flächendeckend freiheitsbeschränkenden“" (S. 2) staatlichen Maßnahmen, die das soziale Leben in den meisten gesellschaftlichen Bereichen, in Schulen, Kindergärten und Kitas, in Wirtschaftsbetrieben und Produktionsstätten, in Restaurants, Sportvereinen und Freizeitclubs abrupt zum Erliegen brachten. Gerechtfertigt waren diese Eingriffe in Grund- und Freiheitsrechte aus der Sicht des Ethikrates durch eine Abwägung konkurrierender moralischer Güter, die auch die Grundprinzipien der Solidarität und Verantwortung aller Bevölkerungsgruppen, nicht nur der unmittelbaren Risikoträger, einbezieht. Ausdrücklich betont die Stellungnahme, dass eine Strategie des „Laufenlassens“, die allein auf die rasche Verbreitung des Virus und eine ausreichende „Durchseuchung“ der Bevölkerung („Herdenimmunität“) setzt, angesichts der unvermeidlichen Mortalitätsrate unverantwortlich gewesen wäre. Insofern billigt der Ethikrat die politische Vorzugsentscheidung der 
staatlichen Akteure, die dem Gesundheitsschutz und den Maßnahmen zur Lebensrettung besonders vulnerabler Gruppen in Krisenzeiten die oberste Priorität staatlichen Handelns zusprachen.

Nur kurz erwähnt wird eine alternative Strategie, die eventuell den Übergang in die spätere Lockerungsphase, die gesellschaftliches Leben wieder ermöglichen soll, als Zusatzstrategie zur weiteren Risikoverminderung begleiten könnte. „Anders zu beurteilen ist möglicherweise ein Vorgehen, das eine solche Strategie mit einem weitreichenden abschirmenden Schutz vulnerabler Gruppen verbindet." (S.2) Doch warnt der DER zugleich davor, dass gerade der Gruppe der Senioren und Hochbetagten, die in Pflegeeinrichtung ohnehin besonders zurückgezogen leben, durch strengere Kontaktsperren psychische Schäden (Einsamkeit, Isolation, depressive Erkrankungen) drohen, die ebenfalls erhebliche Auswirkungen auf ihre gesundheitliche Situation haben.

In ihrer abschließenden ethischen Bewertung der Gesamtstrategie zur Eindämmung der Corona-Pandemie hebt die Stellungnahme zwei Gesichtspunkte hervor: Auch in Katastrophenzeiten kann es nicht allein darum gehen, das unmittelbare Ziel der getroffenen Maßnahmen, die Senkung der Zahl von Neuinfektionen und die Reduzierung der Sterbeziffer, mit allen geeignet erscheinenden Mitteln zu erreichen. Zugleich hat der Staat auch angesichts singulärer Notlagen Sorge dafür zu tragen, dass die Fundamente der Rechtsordnung gewahrt bleiben. Damit hängt zweitens die Notwendigkeit einer ständigen Überprüfung zusammen, ob die Einschränkungen der Grund- und Freiheitsrechte der Bevölkerung noch verhältnismäßig sind. Die Berücksichtigung eines Zeitfaktors bei der geforderten komplexen Güterabwägung ergibt sich aus der Architektonik der Freiheitsrechte, nach der Gesundheits- und Lebensschutz nur zeitweilig die oberste Priorität gegenüber allen anderen Rechten genießen dürfen. „Auch der gebotene Schutz menschlichen Lebens gilt nicht absolut. Ihm dürfen nicht alle Freiheits- und Partizipationsrechte sowie Wirtschafts-, Sozial- und Kulturrechte bedingungslos nach- bzw. untergeordnet werden. Ein allgemeines Lebensrisiko ist von jedem zu akzeptieren." (S. 5)

\section{Der ethische Ernstfall: Entscheidungsregeln der Triage-Situationen}

Den zweiten Schwerpunkt der Stellungnahme bilden Kriterien zur Bewältigung dilemmatischer Entscheidungssituationen, die dann auftreten können, wenn sich selbst die flächendeckenden und eingriffsintensiven Schutzmaßnahmen des Grundszenarios als nicht ausreichend erweisen sollten. In der Rückschau auf die Anfangsphase der Krise zeigt sich, dass 
das deutsche Gesundheitssystem zu jedem Zeitpunkt ihres bisherigen Verlaufs weit von der befürchteten Überlastung entfernt war. Viele Krankenhäuser hatten durch die Umwidmung anderer intensivmedizinischer Behandlungsressourcen und die Bereitstellung zusätzlicher Betten ihre Kapazität so erweitert, dass sie eher über eine mangelnde Auslastung (auch weil bereits geplante Behandlungen anderer Patienten verschoben wurden) klagten. Doch beim Erscheinen des DER-Papiers schien das Worst-CaseSzenario, das durch den Lockdown unbedingt verhindert werden sollte, noch nicht endgültig gebannt. Für diese damals im Bereich des Möglichen liegende Situation, wenn tatsächlich nicht mehr ausreichend intensivmedizinische Ressourcen für alle behandlungsbedürftigen Infizierten zur Verfügung stünden, waren die vom DER vorgeschlagenen ethischen Entscheidungshilfen gedacht. Derartige Triage-Konstellationen werfen schwerwiegende Fragen der Verteilungsgerechtigkeit auf, in denen es für die Betroffenen um Leben und Tod gehen kann. Deshalb kann es, so betont die Stellungnahme, „für manche dieser Konstellationen [...] keine rechtlich und ethisch umfassend befriedigende Lösung“ (S.3) geben.

Für das ärztliche Ethos stellt die Notwendigkeit, unter den Bedingungen von Unsicherheit knappe Behandlungsressourcen bestimmten Patienten zuzuordnen und sie damit anderen vorzuenthalten, eine besondere Herausforderung dar. Diese ist dadurch bestimmt, dass die Maxime, das Wohl des einzelnen Kranken als oberste Richtschnur aller ärztlichen Maßnahmen anzusehen, der Ergänzung durch eine Berücksichtigung der allgemeinen Gefahrenlage und der an sich ebenfalls erforderlichen Gesundheitsfürsorge für die große Zahl anderer als an Corona erkrankter Patienten bedarf, für die keine weiteren Behandlungsplätze mehr zur Verfügung stehen. Die mit dieser Öffnung des ärztlichen Pflichtenkreises verbundene Notwendigkeit von Priorisierungsentscheidungen kann das medizinische Personal vor unlösbare Dilemmata führen, in denen fundamentale Prinzipien des Rechts und der Ethik miteinander kollidieren. Die Stellungnahme des Ethikrats beschreibt diesen Konflikt in aller Schärfe, gibt aber keine konkreten Kriterien an die Hand, an denen sich die Entscheidungsfindung orientieren könnte. Offenbar war über eine Liste positiver Entscheidungskriterien unter den Mitglieder des Ethikrates keine Verständigung zu erzielen, so dass sich die Stellungnahme auf Erwägungen allgemeiner Art beschränken musste.

Diese Überlegungen heben in aller wünschenswerten Deutlichkeit zwei Grundsätze hervor, in denen Rechtsordnung und Ethik (zumindest eine autonomiebasierte Ethik, die dem individuellen Rechtsschutz aufgrund der Menschenwürde oberste Priorität einräumt) übereinstimmen. Dies sind die beiden Grundsätze der Lebenswertindifferenz und des Instrumentali- 
sierungsverbotes. Das erste Prinzip untersagt jede Bewertung des menschlichen Lebens nach Kriterien der Nützlichkeit, des Alters, des sozialen Ranges, der ethnischen Zugehörigkeit oder des Geschlechts. Dieses fundamentale Bewertungsverbot ergibt sich unmittelbar aus dem Gleichheitsgrundsatz der Verfassung, demzufolge jedes menschliche Leben den gleichen Schutz genießt. Damit ist jede Abwägung gemäß einem utilitaristischen Nutzenkriterium - etwa im Sinne einer höheren Zahl geretteter Patienten oder der Maximierung gewonnener Lebensjahre - untersagt. Zugleich darf in Situationen, in denen Leben gegen Leben steht, niemand als Mittel zum Zweck der Lebensrettung anderer benutzt werden. Selbstverständlich muss es das Ziel aller getroffenen Maßnahmen sein, so viele Menschenleben wie möglich zu retten. Doch muss sich die gewählte Vorgehensweise zwingend innerhalb des durch die Grundrechte aller Beteiligten gezogenen verfassungsrechtlichen Rahmens bewegen, die auch in Notsituationen niemals außer Kraft gesetzt werden können. „Auch persönliche ethische Überzeugungen, die etwa eine reine Ergebnisorientierung und mit ihr die unbedingte Maximierung der Zahl geretteter Menschenleben fordern mögen, können ein Handeln, das die skizzierten Grenzen des Verfassungsrechts überschritte, nicht rechtfertigen." (S. 4)

Wie in der medizinethischen Fachliteratur üblich, unterscheidet die Stellungnahme zwei Fallgruppen, in denen Priorisierungsentscheidungen getroffen werden müssen: eine Triage bei Ex-ante-Konkurrenz und die Triage bei Ex-post-Konkurrenz. In seiner Begründung, warum TriageEntscheidungen im ersten Fall möglich sind, bleiben die Ausführungen der Stellungnahme allerdings vage und unbestimmt. Über den Grundsatz hinaus, dass niemand zu Unmöglichem verpflichtet sein kann, wird nur gefordert, dass die Entscheidung aus ethischer Sicht nach wohlüberlegten, einheitlichen und transparenten Kriterien erfolgen sollte. Welche Regeln dafür in Frage kommen, erfährt der Leser hingegen nicht. Die Rechtsordnung kann für derartige dilemmatische Situationen ohnehin keine positiven Entscheidungshilfen geben. An dieser Stelle wären weitere ethische Überlegungen darüber notwendig gewesen, warum Priorisierungsentscheidungen vor Aufnahme der Behandlung erlaubt und oftmals sogar geboten sein könnten. Diese widersprechen nämlich nicht dem strikten Verbot, den Wert des Lebens eines Menschen im Vergleich zu dem eines anderen $\mathrm{zu}$ beurteilen, etwa indem das Leben einer 45 -jährigen Mutter dem eines 85 -jährigen Rentners vorgezogen würde. Beurteilt wird bei einer TriageEntscheidung nicht der Wert der in Konkurrenz zueinander stehenden behandlungsbedürftigen Personen, sondern die Aussicht darauf, dass eine mögliche Behandlung ihr erhofftes Ziel auch tatsächlich erreichen kann, 
also mithin die medizinische Prognose, die häufig unterschiedlich ausfallen wird.

Hingegen darf das Alter als solches kein Ausschlusskriterium sein, wohl aber können in höherem Alter gehäuft auftretende Vorerkrankungen dazu führen, dass ein älterer Patient im Vergleich zu einem jüngeren eine geringere Heilungschance aufweist. Die psychologische Belastung für das ärztliche Personal, die mit derartigen Entscheidungen verbunden ist, bleibt zwar unzumutbar hoch, doch ist die Bewertung von Heilungsaussichten und der Vergleich von Behandlungsoptionen für verschiedenen Patienten durchaus eine Aufgabe, in der medizinisches Wissen zur Anwendung kommt. Aus ethischer Sicht muss die Forderung lauten, dass TriageEntscheidungen nur nach medizinischen Kriterien, sofern diese eine begründete Entscheidungsbasis erlauben, erfolgen dürfen. Wenn bei einem Patienten die begründete Aussicht auf eine bessere Heilungsprognose als bei einem anderen besteht, erscheint es ethisch vertretbar, ihm diese zukommen zu lassen, auch wenn das für den Patienten mit den schlechteren Aussichten bedeutet, dass ihm nicht geholfen werden kann. Offen lässt der DER auch die Frage, ob Personen, die sich bei der Ausübung ihres ärztlichen oder pflegerischen Berufs infizieren (bei denen die Erkrankung oft einen besonders schweren Verlauf nimmt), vorrangig behandelt werden dürfen. Das würde zwar die Grundregel durchbrechen, Priorisierungen nur nach medizinischen Kriterien vorzunehmen. Doch ließen sich zugunsten derartiger Entscheidungen Gerechtigkeitsüberlegungen anführen, da sich dieser Personenkreis einem wesentlich höheren Infektionsrisiko aussetzen muss, um bereits Erkrankten helfen zu können. Auch liegt es im Interesse aller aktuell und künftig Infizierten, die Aufrechterhaltung der medizinischen Versorgung durch die möglichst rasche Rückkehr der Betroffenen an ihren Arbeitsplatz im Krankenhaus sicherzustellen.

Schwieriger sind die Fälle zu beurteilen, in denen es zu einer Ex-postKonkurrenz unter mehreren Patienten kommen kann. Darf die lebenserhaltende Behandlung eines Patienten - angenommen es handle sich um den in der öffentlichen Debatte um die Stellungnahme des Ethikrats viel zitierten 85-jährigen Rentner - beendet werden, um mit den medizinischen Behandlungskapazitäten das Leben eines anderen Menschen - etwa das einer 45-jährigen Mutter - zu retten, der sich zu einem späteren Zeitpunkt infiziert? Aus rechtlicher Sicht wäre dies - anders als bei den Exante-Konstellationen - als „Töten durch Unterlassen“ zu qualifizieren. ${ }^{1}$ Aber auch moralisch liefe es auf eine unerlaubte Instrumentalisierung hi-

1 Vgl. Rönnau/Wegner, Grundwissen - Strafrecht: Triage, 403-407, bes. 405f. 
naus, würde die laufende Behandlung des ersten Patienten zugunsten der Rettung eines anderen beendet. Die Stellungnahme des DER spricht sich nicht eindeutig für die Geltung des sogenannten Windhund-Prinzips aus, wonach derjenige, der zuerst an das medizinische Gerät angeschlossen wird, diesen zeitbedingten Vorteil solange behält, als die Fortführung der Behandlung für ihn medizinisch indiziert ist. Das Papier beschränkt sich auf die Feststellung, dass die Beendigung einer lebenserhaltenden Maßnahme mit ausreichender Erfolgsprognose niemals „objektiv rechtens“ sein kann. (S. 4)

Dadurch gerät der DER in die unangenehme Situation, der Stellungnahme der Leopoldina, die derartige Entscheidungen nicht in jedem Fall für unzulässig hält, indirekt widersprechen zu müssen. Deshalb hält auch der DER eine andere individuelle ärztliche Entscheidung für moralisch immerhin tolerabel, auch wenn ihr eine rechtliche Handlungslegitimation fehlt. „Hier können Grenzsituationen entstehen, die für das behandelnde Personal seelisch kaum zu bewältigen sind. Wer in einer solchen Lage eine Gewissensentscheidung trifft, die ethisch begründbar ist und transparenten - etwa von medizinischen Fachgesellschaften aufgestellten - Kriterien folgt, kann im Fall einer möglichen (straf-)rechtlichen Aufarbeitung des Geschehens mit einer entschuldigenden Nachsicht der Rechtsordnung rechnen." (S.4) Ob sich die einzelnen ärztlichen Entscheider durch den bloßen Hinweis auf das Vorliegen eines rechtlichen Strafausschließungsgrundes in ihrer Urteilskompetenz tatsächlich bestärkt fühlen, darf allerdings bezweifelt werden.

In ihrer Analyse des Vergleichs der Behandlungsbedürftigkeit mehrerer Patienten im Rahmen einer Ex-post-Triage-Situation geht die Stellungnahme des DER davon aus, dass die lebenserhaltende Behandlung für den bereits an das medizinische Gerät angeschlossenen Patienten weiterhin indiziert ist. In diesem Fall errichtet das rechtliche Verbot, die begonnene Behandlung vorzeitig abzubrechen, eine Grenze, die auch in ethischer Hinsicht Beachtung verlangt; solange die Fortführung der begonnenen Behandlung nach medizinischen Kriterien indiziert ist, weil noch Aussicht besteht, dass sie ihr Ziel erreichen kann, bleibt jede andere Lösung durch das Instrumentalisierungsverbot untersagt. Für die nachfolgenden Patienten, die aufgrund fehlender medizinischer Kapazitäten oder wegen der Belegung aller vorhandenen Behandlungsplätze nicht mehr versorgt werden können, erscheint es besonders bitter, von einer an sich möglichen und in den meisten Fällen erfolgversprechenden Therapie wegen ihrer im Augenblick fehlenden Verfügbarkeit ausgeschlossen zu bleiben. Doch muss diese Lage, deren Auftreten man einem tragischen „Zufall“ oder dem „Schick- 
sal“ zuschreiben kann, hingenommen werden, da sie sich auf eine moralisch und rechtlich vertretbare Weise nicht ändern lässt.

Die Prämisse, dass die Behandlung des ersten Patienten weiterhin indiziert ist, bedarf jedoch während des Therapieverlaufs einer ständigen Überprüfung. Die Konstellation, dass eine Behandlung durch die zunehmende Verschlechterung des Gesamtzustandes eines Patienten unverhältnismäßig wird und aus diesem Grund abgebrochen werden darf, kann sich auch im Rahmen einer intensivmedizinischen Anti-Corona-Unterstützungsbehandlung ergeben. ${ }^{2}$ Wenn es angebracht erscheint, einen Therapieziel-Wechsel hin zu einer palliativmedizinischen Versorgung ins Auge zu fassen, wird der Behandlungsplatz frei für einen Nachfolge-Patienten. Diese grundsätzlich bei jeder medizinischen Behandlung denkbare Situation ist aber nur dann gegeben, wenn die Fortführung der Behandlung aus der Sicht des aktuell behandelten Patienten unverhältnismäßig geworden ist. Diese Annahme ist aber nicht schon dadurch erfüllt, dass weitere Anwärter auf den Behandlungsplatz in der Warteschlange nachrücken. Ebenso kann es als legitim angesehen werden, immer ein Notfallbett freizuhalten, um sich Behandlungsoptionen in einem Worst-Case-Szenario zu bewahren, in denen ein junger Patient ohne Vorerkrankungen mit guter Genesungsprognose zu einem Zeitpunkt kommt, an dem alle Behandlungsplätze belegt sind. Auf derartige Details geht die Stellungnahme des DER jedoch nicht ein, da sie nur verlangt, dass eventuell notwendige Triage-Entscheidungen nach transparenten und einheitlich angewandten Regeln erfolgen sollen.

\section{Die Notwendigkeit einer Öffnungsperspektive}

Als dritten Fragenkomplex erörtert die Stellungnahme die Notwendigkeit einer „Öffnungsperspektive“ (S. 6), um die Bereitschaft der Öffentlichkeit, die verfügten Freiheitsbeschränkungen im Geiste solidarischer Zusammengehörigkeit mitzutragen, über einen längeren Zeitraum hinweg zu erhalten. Damit ist weder eine einfache Rückkehr zum status quo ante, noch die Zeit danach gemeint, die erst anbricht, wenn die Corona-Krise vollständig überwunden ist. Vielmehr umfasst der Begriff die Lockerungen und allmählichen Aufhebungen der zu Beginn der Krise getroffenen Schutzmaßnahmen, die schon bald nach der Überwindung (oder dem Ausbleiben) der ersten Infektionswelle angezeigt sind. Die allmähliche Renormalisierung des gesellschaftlichen Lebens muss nach Auffassung des DER von

2 Vgl. Heinemann/Proft u. a., Covid-19. Ethische Empfehlungen, 32. 
einer intensiven gesellschaftlichen Debatte darüber begleitet werden, welches Maß an Lebensrisiken die Mehrheit zu tolerieren bereit ist, und welche längerfristigen Einschränkungen sie in welchen Bereichen zur Minimierung dieser Risiken mittragen möchte.

Angesichts der massiven gesellschaftlichen ökonomischen und kulturellen Langzeitfolgen, die der Lockdown für den Lebensstand der Gesellschaft haben wird, ist es nach Ansicht des DER somit schon auf dem Höhepunkt der Krise erforderlich, eine geordnete Rückkehr zu „normalen“ gesellschaftlichen Aktivitäten und zu regulären wirtschaftlichen Unternehmungen ins Auge zu fassen, um die materiellen, kulturellen und psychosozialen Schäden möglichst gering zu halten. Besonders hebt der DER die Notwendigkeit hervor, die ökonomische Basis der Gesellschaft zu erhalten. Dabei geht es nicht nur darum, die Interessen des „Kapitals“ zu berücksichtigen, wie im öffentlichen Diskurs häufig mit herabsetzendem Unterton vermerkt wird. Denn um die Bewältigung der durch die Corona-Krise entstandenen Lage dauerhaft absichern zu können, muss nicht nur die Handlungsfähigkeit des Staates und seiner Verwaltungsinstanzen gewährleistet sein - wie gut diese während der Krise funktionierten, konnte die deutsche Bevölkerung zu ihrem Erstaunen und mit positiver Überraschung registrieren. Vielmehr bedarf es auch einer funktionierenden marktwirtschaftlichen Ordnung, um die erforderlichen Wohlfahrtsgewinne langfristig zu erarbeiten.

Besonders betont der Ethikrat, dass die wirtschaftliche Existenz vor allem von Kleinunternehmern und Selbständigen gefährdet ist, die in bestimmten Branchen wie der Hotellerie, dem Gastgewerbe oder dem Kulturbereich tätig sind, und das Ausbleiben regelmäßiger Einnahmen nicht lange überleben können. Aber auch ein Zusammenbruch des marktwirtschaftlichen Gesamtsystems ist für den Fall zu befürchten, dass zu viele Unternehmen der mittelständischen Industrie aufgrund einer geringen Eigenkapitaldecke Insolvenz anmelden müssen. Die enge Verflechtung der industriellen Produktion und die langen Lieferketten erhöhen in Krisenzeiten die Risiken für das Gesamtsystem, da Ausfälle an einer Stelle entsprechende Wirkungen in nahezu allen anderen Bereichen nach sich ziehen. Zudem erinnert die Stellungnahme an das Schicksal vieler Menschen, die aufgrund der Krise ihren Arbeitsplatz verlieren oder - besonders auch in prekären Lebensverhältnissen - dauerhaft auf Kurzarbeit umstellen müssen.

Die Dimension der genannten Schäden in den unterschiedlichen Lebensbereichen zwingt nach Ansicht des DER dazu, die Erforderlichkeit und Angemessenheit der ergriffenen Maßnahmen ständig zu überprüfen. Wenn das Ziel des Lockdowns, eine mögliche Überlastung des Gesundheits- 
systems abzuwenden, erreicht ist und die Reduplikationsrate, also die Zahl der Infizierten, die einen anderen Menschen ansteckt, dauerhaft unter 1 liegt, ist eine Aufrechterhaltung der Maßnehmen nicht mehr gerechtfertigt. „Wenn und soweit dieser Zustand erreicht wird, ist der schrittweise und epidemiologisch evaluierte Abbau der Restriktionen nicht nur möglich, sondern geboten." (S. 6) Für die Zeit nach der Überwindung der Krise, wenn wirksame Medikamente und verträgliche Impfstoffe vorliegen werden, gibt der Ethikrat schließlich Empfehlungen zur Verbesserung des Gesundheitswesens und dem Ausbau von Testkapazitäten zur Diagnostik, die dafür sorgen sollen, dass man im Falle der Wiederholung ähnlicher Pandemien in Zukunft besser gerüstet sein wird. Viele der anfangs in Deutschland schmerzlich vermissten Maßnahmen waren an sich in den Notfallplänen vorgesehen, die von den zuständigen Ministerien regelmäBig überarbeitet werden sollen, aber im politischen Normalbetrieb oft vernachlässigt werden und auch in der Öffentlichkeit nur wenig Aufmerksamkeit finden.

Beiläufig erwähnt die Stellungnahme, dass die verfügten Einschränkungen des sozialen Lebens verschiedene Altersgruppen in unterschiedlichem Maße treffen und somit auch Fragen der Generationengerechtigkeit aufwerfen. Jüngere Menschen oder solche, die eine Infektion bereits überstanden und dadurch (wahrscheinlich) eine persönliche Immunität aufgebaut haben, mögen vielleicht geneigt sein, für sich höhere Risiken zu akzeptieren, da sie sich selbst als weniger gefährdet einschätzen. Dies kann sogar bei Angehörigen von Hochrisikogruppen der Fall sein, die persönlich ein höheres Risiko in Kauf zu nehmen gewillt sind, und deshalb die vorgeschriebenen Schutzmaßnahmen zugunsten einer größeren Freizügigkeit durchbrechen möchten. Gegenüber solchen persönlichen Erwägungen ruft der DER in Erinnerung, dass die eigenen Entscheidungen und die eigene Lebensführung erhebliche Auswirkungen auf die Entscheidungen und die Lebensführung anderer Menschen haben. Dies gilt besonders in Krisenzeiten, in denen keine eingespielten Verhaltensmuster verfügbar sind, sondern vom einzelnen die Bereitschaft gefordert ist, Einschränkungen auch dann verantwortlich mitzutragen, wenn sie nicht von allen eingesehen werden. Dies gilt auch für die Solidarität zwischen den Generationen, wenn in einer Gefährdungslage die persönliche Betroffenheit durch potenzielle Risiken umgekehrt verteilt ist. Die Angehörigen der älteren Generation, zu deren Schutz Kinder und Jugendliche in Zeiten der Corona-Krise überproportional und ungefragt herangezogen werden, können darin einen gerechten Ausgleich für die Aufbauleistungen sehen, durch die sie in den vergangenen Jahrzehnten den derzeitigen Wohlstand geschaffen haben. Sie werden aber schon bald Gelegenheit bekommen, auch 
ihrerseits aus Solidarität mit den Jüngeren Einschränkungen ihres Lebensstils hinzunehmen - spätestens, wenn es in der Umwelt- und Klimapolitik darum geht, künftige Entwicklungen abzumildern, von denen sie zu ihren Lebzeiten nicht mehr betroffen sein werden.

\section{Literatur}

Deutscher Ethikrat: Solidarität und Verantwortung in der Corona-Krise. Ad-hocEmpfehlung, Berlin 2020, in: https://www.ethikrat.org/fileadmin/Publikationen /Ad-hoc-Empfehlungen/deutsch/ad-hoc-empfehlung-corona-krise.pdf [28.05.2020].

Heinemann, Thomas/Proft, Ingo et al.: Covid-19. Ethische Empfehlungen über Beginn und Fortführung einer intensiv-medizinischen Behandlung bei nicht ausreichenden Behandlungskapazitäten, Vallendar 2020, in: https://www.pthv.de/fil eadmin/user_upload/ALTE_ORDNER/PDF_Theo/Ethik-Institut/\%C3\%96ffentl ichkeitsarbeit/Stellungnahme_Covid-19_Heinemann_Proft.pdf [28.05.2020].

Rönnau, Thomas/Wegner, Kilian: Grundwissen - Strafrecht: Triage, in IuS 5 (2020) 403-407, insbes. 405f. 
